\title{
(2) \\ La orientación psicoeducativa en las instituciones de educación superior como estrategia de acompañamiento al aprendizaje
}

Psychoeducational orientation in higher education institutions as a learning accompaniment strategy

A orientação psicoeducacional em instituições de ensino superior como estratégia de acompanhamento da aprendizagem

Francisca Toledo-Candia

Universidad Metropolitana de Ciencias de la Educación (Chile)

https://orcid.org/0000-0002-6705-4025

D0I: https://doi.org/10.35622/j.rep.2021.04.005

Recibido 04/06/2021/ Aceptado 16/11/2021

RESUMEN. La educación superior ha experimentado una expansión a nivel global. En Chile, esta ampliación supone desafíos a las instituciones de educación superior quienes deben desarrollar programas de acompañamiento al aprendizaje de sus estudiantes. En este artículo, a partir de la experiencia de orientación psicoeducativa en estudiantes de educación superior, se propone un modelo de orientación psicoeducativa como estrategia de acompañamiento y desarrollo de habilidades de aprendizaje metacognitivo, se consideran las necesidades, fortalezas y carencias de los y las estudiantes, asociadas a sus conocimientos previos, disposiciones psico-sociales, actitudinales y valóricas.

PALABRAS CLAVE: orientación psicoeducativa, estrategia de acompañamiento, aprendizaje, educación superior.

ABSTRACT. Higher education has expanded globally. In Chile, this expansion challenges higher education institutions to develop support programs for their students' learning. In this article, based on the experience of psychoeducational guidance in higher education students, a model of psychoeducational guidance is proposed as a support strategy and development of metacognitive learning skills, considering the needs, strengths, and shortcomings of the students, associated with their previous knowledge, psychosocial, attitudinal, and value dispositions.

KEYWORDS: psychoeducational guidance, support strategy, learning, higher education.

RESUMO. 0 ensino superior se expandiu globalmente. No Chile, esta expansão representa desafios para as instituições de ensino superior que devem desenvolver programas de apoio à aprendizagem de seus alunos. Neste artigo, com base na experiência de orientação psicoeducacional em alunos do ensino superior, é proposto um modelo de orientação psicoeducacional como estratégia de apoio e desenvolvimento de habilidades metacognitivas de aprendizagem, considerando as necessidades, potencialidades e deficiências dos alunos, associadas às suas anteriores. disposições de conhecimento, psicossociais, atitudinais e de valor.

PALABRAS CLAVE: orientação psicoeducacional, estratégia de apoio, aprendizagem, ensino superior 


\section{INTRODUCCIÓN}

La educación superior ha experimentado una expansión a nivel global. En Chile, esta ampliación presenta una realidad dual: por un lado, como política pública reconoce la universalización de la educación superior en tanto logro positivo; por otro lado, adquiere ribetes negativos por la masificación imbricada con la mercadización (Jarpa \& Rodríguez, 2017, p. 328). A esto se suma una serie de políticas e iniciativas de equidad en el sistema de acceso desarrolladas por el Ministerio de Educación (MINEDUC) y las Universidades. Desde el MINEDUC se han implementado medidas que se centran a) en el aseguramiento de la calidad de la educación superior, b) en el aumento del acceso por medio del sistema de gratuidad, becas, créditos y c) en favorecer el acceso con equidad, evitando la deserción a través del desarrollo de iniciativas de nivelación y de acompañamiento al trayecto formativo.

Dentro de las medidas implementadas para favorecer el acceso equitativo, encontramos la diversificación y modificación de los mecanismos de admisión como la introducción del ranking de notas de enseñanza secundaria, como criterio de admisión y asignación de becas, la creación del Programa de Acompañamiento y Acceso Efectivo a la Educación Superior (PACE), los programas propedéuticos, los cupos de equidad, las becas indígenas y los programas de equidad de género en las carreras de ingeniería. Lo anterior, ha llevado a un incremento de la participación en la educación superior de grupos sociales antes excluidos (Espinoza \& González, 2015) como minorías étnicas y sectores de bajos ingresos. Esto ha diversificado el perfil de ingreso, permitiendo un aumento de la matrícula de estudiantes que son la primera generación de sus familias en acceder a la educación superior.

Por otra parte, la transición entre la enseñanza secundaria y la educación superior considera una gran cantidad de dificultades para los estudiantes, pues como plantea Schiefelbein \& Zúñiga (2000) supone

una continuidad-discontinuidad con la educación recibida previamente, que suele ser una tarea difícil para muchos de los nuevos estudiantes, ya que hay algunos hábitos que deben cambiar, tales como las estrategias de aprendizaje que fueron útiles en la etapa previa, pero que en la nueva deben modificar (p.8). 
Si a lo anterior, agregamos la escasez de recursos que obligan a muchos estudiantes a trabajar, los largos trayectos de desplazamiento, la falta de redes de apoyo y el bajo capital cultural producto de las desigualdades sociales, culturales y económicas. La tarea de adaptación a la educación superior es aún más compleja, afectando negativamente el rendimiento académico, transformándose en una situación de fracaso académico que está condicionada previamente (Garbanzo, 2007). Esto plantea nuevos desafíos a las instituciones de educación superior quienes deben repensar sus procesos formativos en función de las necesidades de la diversidad de estudiantes para asegurar la retención y el logro académico de estos.

A nivel internacional, las tasas de abandono y de retención de estudiantes, así como el éxito académico constituyen indicadores relevantes en la educación superior, debido a que para lograr una educación superior de calidad y con equidad, es importante garantizar la permanencia, el desempeño y las posibilidades de que los estudiantes obtengan buenos resultados en sus estudios. Es por lo anterior, que estos índices son monitoreados por diversos organismos como la Organización para la Cooperación y el Desarrollo Económico (OCDE), el Banco Mundial, Instituto Internacional para la Educación Superior en América Latina y el Caribe (IESALC) y el Centro Interuniversitario de Desarrollo (CINDA).

Desde el Estado de Chile, en el marco de un convenio de préstamos entre el Gobierno de Chile y el Banco Internacional de Reconstrucción y Fomento (BIRF), se ha promovido a través de diversos instrumentos la generación de estrategias de retención de estudiantes en las instituciones de educación superior.

En este contexto, el Programa de Mejoramiento de la Calidad y Equidad en la Educación Superior (Mecesup) ha intentado instalar la preocupación por la deserción en las instituciones, financiando acciones y programas para nivelar las oportunidades de aprendizaje de los estudiantes, y fomentar su permanencia en el sistema (Llanos y Martínez, 2018, p.4).

Las universidades chilenas para contribuir al aprendizaje de los estudiantes, y por consecuencia, a mejorar los índices de aprobación de las asignaturas, las tasas de permanencia y de titulación oportuna de los estudiantes, implementan estrategias y 
acciones de acompañamiento como formas de apoyo a los estudiantes que presentan dificultades durante su trayecto formativo. Estas acciones se implementan como programas de consejerías, de tutorías, de asesorías y de orientación. Asimismo, forman parte de procesos continuos de diagnóstico y evaluación de necesidades educativas de los estudiantes que son planificados en las diferentes instituciones.

En este contexto, en el Centro de Acompañamiento al Aprendizaje de la Universidad Metropolitana de Ciencias de la Educación (CAA-UMCE), se incorpora, como proceso de acompañamiento a los aprendizajes de estudiantes, la orientación psicoeducativa, con el objetivo de abordar los problemas afectivos y sociales que influyen en el aprendizaje y en la trayectoria académica de los estudiantes, contribuyendo al desarrollo y potenciando diversas habilidades que son necesarias durante el proceso formativo universitario. Para generar este acompañamiento revisamos la literatura especializada sobre la temática, dándonos cuenta que la mayoría de los artículos sobre orientación psicoeducativa se centraban en estudiantes de educación primaria y secundaria, y estaban pensados para la institución escolar.

El conocimiento acumulado sobre la experiencia en la educación superior es incipiente, siendo que es necesaria su construcción. Esto debido a que existen particularidades ligadas a la educación superior que hacen de la orientación psicoeducativa un proceso con características propias. El estudiante de educación superior se encuentra en una etapa de desarrollo psicológico, social y cognitivo diferente que un estudiante de primaria y secundaria. Asimismo, sus problemáticas y necesidades son diferentes por las mismas continuidades/discontinuidades que se plantean anteriormente. De esta manera, resulta relevante comunicar nuestra experiencia de orientación psicoeducativa en estudiantes de educación superior y avanzar hacia un modelo de orientación en la universidad como estrategia de acompañamiento al aprendizaje.

\section{Una experiencia de orientación psicoeducativa desarrollada en una universidad con sello pedagógico}

La UMCE es una universidad estatal chilena que posee 22 carreras de pregrado (19 corresponden a formaciones pedagógica) y una matrícula de 4853 estudiantes (2967 
mujeres y 1886 hombres), compuesta principalmente por estudiantes de pedagogía que provienen mayoritariamente de dependencias municipales y particulares subvencionadas. A su vez, la mayoría de éstos pertenecen a sectores vulnerables. Esto se ve reflejado en la gran cantidad de estudiantes que poseen algún beneficio del Estado. Según el anuario estadístico de la UMCE, el año 2018 había 2.148 estudiantes con gratuidad y 1605 estudiantes con beca de arancel MINEDUC, es decir, el 87,1\% de sus estudiantes poseía algún tipo de beneficio.

La universidad tiene como misión servir a los propósitos de la educación en todas sus manifestaciones, atendiendo, especialmente, la formación profesional docente- inicial y continua- para todos los niveles, sectores y modalidades del sistema educacional chileno (UMCE, 2016, p.8). En su modelo educativo destaca una formación orientada a la construcción de saberes a través de la articulación de la teoría y la práctica. De esta manera, el estudiante de la UMCE va construyendo su identidad profesional en la especialidad y en la intervención de contextos reales desde su inicio (UMCE, 2016, p.23).

El año 2017, en el marco del proyecto MECESUP UMC1406, se crea el CAA-UMCE, cuyo objetivo es desarrollar procesos de acompañamiento al aprendizaje diseñando, articulando, coordinando y evaluando iniciativas que favorezcan el trayecto formativo de los estudiantes de la UMCE. Desde el CAA-UMCE se implementan una serie de acciones de acompañamiento al aprendizaje durante todo el trayecto formativo del estudiante de pregrado de la Universidad, para así trabajar sus debilidades e intentar desarrollar al máximo sus potencialidades. En este contexto, las acciones de acompañamiento son un proceso intencionado y un espacio de posibilidades para la (re)construcción de conocimientos y (re)creación de nuevas prácticas que permiten potenciar los aprendizajes de los estudiantes. Las acciones implementadas para su desarrollo se consideran las particularidades de los estudiantes, su diversidad, sus fortalezas y sus necesidades, por lo que adquiere relevancia la caracterización y el diagnóstico que ejecutamos previamente y que se va retroalimentando durante todo el periodo en que se ejecuta la acción.

En el contexto de una universidad con sello pedagógico, la acción de acompañamiento se orienta al proceso formativo de los estudiantes como futuros docentes en las áreas cognitiva, socioemocional y de fortalecimiento profesional. Es decir, las acciones se dirigen al desarrollo de competencias de acción profesional y socioemocionales, entendiendo una competencia como el conjunto de conocimientos, capacidades, habilidades y actitudes necesarias para realizar actividades diversas 
con un cierto nivel de calidad y eficacia de acción profesional. En el concepto de competencia se integra el saber, saber hacer y saber ser (Bisquerra, 2003, p. 21).

En el ámbito socioemocional, el futuro profesor requiere tener herramientas que le permitan afrontar la presión y el estrés que tienen en las aulas y en las escuelas, aprender a trabajar y relacionarse con estudiantes, colegas y padres, así como también, desarrollar estas competencias en sus estudiantes. De aquí, la importancia de potenciar, en los futuros docentes, una serie de competencias emocionales, es decir, capacidades y disposiciones que facilitan la habilidad para crear, manejar sentimientos y estados de ánimo a partir del conocimiento que poseemos sobre lo que sucede y para ello debemos conocer y reconocer los pensamientos y comportamientos que subyacen (Bisquerra, 2009, p.130).

En esta línea, Barrientos (2016) señala que un docente debe ser social y emocionalmente competente, en otras palabras, debe ser capaz de comunicarse con sus estudiantes, saber afrontar los conflictos dentro de su aula, crear un ambiente cálido y agradable en su sala de clases, tener empatía con sus estudiantes, ser flexible, adaptándose a diferentes cambios, construir relaciones sociales basados en el respeto mutuo y el reconocimiento. Es decir, la competencia emocional estará relacionada con demostrar autoeficacia cuando se expresan emociones en las transacciones sociales (Saarni, 2000).

\section{El modelo de orientación psicoeducativa desarrollado en el CAA-UMCE.}

La orientación psicoeducativa es un proceso de carácter formativo, planificado e intencional, que se concibe como un conjunto de estrategias de apoyo que se ajustan a las necesidades educativas del estudiante. Puede considerarse una relación de ayuda, donde se analiza y reflexiona sobre las necesidades de la persona en un tiempo, espacio y contexto determinado para que pueda crecer personal, académica y profesionalmente. Facilita el desarrollo personal y profesional durante el proceso educativo, favorece la resolución de problemas y prepara a los estudiantes para la acción transformadora (Sánchez, 2017).

De esta manera, podemos decir, que la orientación psicoeducativa posee un carácter preventivo, desarrolla habilidades que permiten asumir una actitud proactiva en el afrontamiento de problemáticas y situaciones que los estudiantes viven cotidianamente. Por lo mismo, es un proceso que posibilita el aprendizaje y el desarrollo de habilidades destrezas para aprender a aprender. Asimismo, la orientación psicoeducativa requiere una preparación profesional debido a que necesita el dominio de ciertos conocimientos teóricos y metodológicos de la pedagogía, la psicología y la psicopedagogía, así como una serie de competencias socioemocionales, como la empatía, la 
escucha activa, la capacidad de dialogo, entre otras (Del Pino, 2013). En concordancia, Arraiz y Sabirón (2012) señalan que ha de ser un profesional competente para que con su pensamiento, su comportamiento y sus intervenciones logre la difícil tarea de favorecer a la persona en un engranaje sistémico no siempre favorable (p.10).

En el CAA-UMCE, para diseñar la acción de acompañamiento, se asume una tendencia integrativa, es decir, una comprensión integral de la educación (Del Pino, 2013, p. 50) y de las problemáticas de la institución de educación superior, asimismo, consideramos a toda la población estudiantil, y no sólo los casos problemáticos o en riesgo. Reconocemos, además, las posibilidades educativas del orientador como figura que puede y debe complementar su trabajo con el docente y otros profesionales (p.51). De esta manera, consideramos que la orientación psicoeducativa la desarrollamos en un contexto determinado y como parte de un sistema de acompañamiento más amplio, por lo que siempre estará en relación con otros profesionales y/o unidades que realicen acciones, así como también, con docentes y/o académicos de la facultad a la que pertenece el estudiante.

La tendencia integrativa se adaptas al CAA-UMCE, otorgándole las particularidades que tiene el contexto de la educación superior en Chile y de una universidad con sello pedagógico que recibe un gran porcentaje de estudiantes proveniente de sectores económicos de bajos ingresos. Se asume un carácter pedagógico, buscando potenciar las competencias transversales (sociales y emocionales) vinculadas al aprendizaje tanto de la propia disciplina como de la práctica profesional docente a partir de la reflexión y el diálogo, donde la persona que aprende es la protagonista favoreciendo al estudiante en sus múltiples facetas y necesidades. La orientación psicoeducativa entonces se constituye como una estrategia cuyo foco es que los estudiantes puedan desarrollar un aprendizaje autónomo. Por lo que cualquier acción que se planifique siempre buscará la autonomía educativa, es decir, se privilegiarán acciones pedagógicas que pueden generar por sí mismos los estudiantes y que se derivan de las necesidades propias de aprendizaje en razón de sus prácticas, sus estrategias, así como de su capital social y cultural (Vera, 2014).

Por otra parte, el modelo de orientación psicoeducativa está pensado en generar una reflexión sobre diferentes contextos de vida del estudiante y trabajar diferentes dimensiones de manera interrelacionada, con el fin de facilitar su desarrollo integral. Es así, que en la orientación psicoeducativa se abordan situaciones afectivas y sociales que influyen en el aprendizaje y en la trayectoria académica, acompañando a estudiantes que presentan dificultades para obtener logros académicos deseados o que han vivenciado situaciones complejas en desarrollo de sus prácticas intermedias y/o profesional. 


\section{Aproximaciones a la noción de aprendizaje y de sujeto que aprende.}

El aprendizaje es entendido como un proceso, eminentemente social, de construcción de conocimiento en interrelación con otros, es decir, un proceso de carácter relacional. Este proceso es permanente a lo largo de toda la vida, tanto de estudiantes como de docentes.

La orientación psicoeducativa estará centrada en el aprendizaje del estudiante y se evaluará constanmente durante todo el proceso, permitiendo que el estudiante tome consciencia de su propio proceso, así como también, retroalimentar la misma acción, modificándola en caso de que no se estén cumpliendo los objetivos propuestos.

Para iniciar el proceso de orientación psicoeducativa, se debe

conocer y comprender a la persona a orientar, porque esta persona vive las circunstancias y condiciones (...) La finalidad de la orientación empieza por comprender al orientado. [Asimismo, resulta relevante realizar] una individualización de los ritmos de aprendizaje, con particular atención a la singularidad en los sentidos ( $y$ dificultades) del aprendizaje (Arraiz \& Sabirón, 2012, p.29).

Es importante considerar las diferentes facetas y contextos del estudiante y favorecer la reflexión y autorreflexión en función de la etapa del ciclo vital en que se encuentre el estudiante.

En el proceso de construcción de aprendizajes de los estudiantes influyen una serie de factores como su condición económica, su capital social y cultural, su motivación, sus expectativas, metas, entre otras cosas. De esta manera, el estudiante será un agente activo a nivel metacognitivo, motivacional y conductual e interactivo (Nieves et. al., 2006, p.3).

La institución universitaria debe propiciar condiciones para que sus estudiantes construyan un conocimiento situado, que es parte y producto del proceso de aprendizaje (Serrano \& Pons, 2011). De este modo, el conocimiento se entenderá como un proceso de construcción genuina del sujeto que se realiza en diálogo con otro, en este caso, la persona que desempeña la acción de acompañamiento. Así, el sujeto que aprende, va modificando sus aprendizajes previos, a partir de las actividades propuestas.

Es importante destacar que en la construcción del conocimiento es un proceso colectivo, en el que tenemos diferentes agentes que participan. Estos son: 
el sujeto que construye el conocimiento, los instrumentos utilizados en la actividad (...), los conocimientos que deben ser construidos, una comunidad de referencia en la que la actividad y el sujeto se insertan, un conjunto de normas de comportamiento que regulan las relaciones sociales de esa comunidad y un conjunto de reglas que establecen la división de tareas en la actividad conjunta (Serrano \& Pons, 2011, p. 10).

Entonces, la orientación psicoeducativa ocurrirá en un contexto donde el o la estudiante, realizan distintas actividades, las cuales permiten la reflexión en torno a un problema o situación real. Reflexiones que son plasmadas luego en un ejercicio que le permite hacer visible los nuevos aprendizajes

La importancia de la práctica metacognitiva en la orientación psicoeducativa

En la universidad y en el desempeño profesional se requiere un dominio autónomo del conocimiento, que

exige una integración del conocimiento teórico (saber decir), de naturaleza declarativa y explícita, y del conocimiento procedimental (saber hacer), que se manifiesta en la acción y es de naturaleza tácita o implícita; en definitiva, exige la integración de la teoría y la práctica.

Para que esa integración pueda tener lugar es preciso fomentar una práctica reflexiva (Pozo \& Pérez, 2009, p.55).

En este sentido, la reflexión sobre la propia acción y el aprendizaje se convierte en un elemento central durante el abordaje de un problema, siendo necesario que el estudiante aprenda a partir de la reflexión en la acción y sobre la propia acción, gestionando sus estudios de forma autónoma. En otros términos, es importante que durante la acción de acompañamiento el estudiante desarrolle un proceso de aprendizaje metacognitivo.

La metacognición es la capacidad que tienen las personas para reflexionar, comprender y controlar su propio pensamiento, actuar y aprender (Chantharanuwong et. al., 2012). Es un proceso cognitivo que puede potenciarse para que la persona sea capaz de controlar su propia actividad de pensamiento planificando, generando seguimiento y evaluación a sus propios aprendizajes.

Según Brown (1987, citado por Chantharanuwong et. al., 2012), la metacognición es un proceso de reflexión sobre los procesos de análisis, síntesis y resolución de problemas del pensamiento durante el aprendizaje. Está relacionado con el conocimiento, la regulación y el control consciente de la actividad cognoscitiva. El desarrollo de las habilidades metacognoscitivas desempeñan un papel 
importante en muchos tipos de actividad cognoscitiva como: persuasión oral, comprensión lectora, la recepción, la atención, la solución de problemas y diversas formas de autocontrol (Flavell, 1985, en Jaramillo \& Simbaña 2014).

La metacognición es relevante en el aprendizaje porque permite resolver problemas en muchas áreas, por ejemplo, la metacognición es central en el desarrollo del pensamiento matématico y científico, así como en la comprensión del lenguaje (Chantharanuwong et. al., 2012). Es por lo mismo que es necesario orientar las prácticas a reflexionar sobre nuestro propio razonamiento y sobre cómo aprendo. En otras palabras, desarrollar consciencia sobre mis propios procesos de aprendizaje, referido

a un "saber qué" acerca de nuestra propia actividad cognitiva (sobre cómo recordamos, aprendemos, comprendemos, razonamos, etc.) (...) [y a] un "saber cómo" que se concreta en un control activo de los recursos disponibles y se traduce en un funcionamiento eficaz en el contexto de una determinada tarea (Pozo \& Pérez, 2009, p.61)

Nuestro modelo de orientación psicoeducativa se orienta hacia el aprender a aprender. En otras palabras, intenta dotar a la persona de aquellas herramientas que le permitan seguir aprendiendo; es decir; aumentar su autonomía y favorecer su autodeterminación (Arraiz \& Sabirón, 2012, p.29). De esta manera, la orientación psicoeducativa está centrada en la persona que aprende y en cómo aprende.

Es importante comprender cómo aprende el estudiante y desde aquí poder potenciar la capacidad de regular su propio proceso de construcción de aprendizajes y aprender de forma autónoma a lo largo de la vida, trabajando la capacidad de aprender a aprender (Nuñez et. al., 2006). Desarrollar un proceso en que los estudiantes son activos y establecen sus propios objetivos que van guiando los aprendizajes de las sesiones de orientación. Estos aprendizajes están en seguimiento constante y autoevaluación constante.

Es importante que las actividades que se desarrollan den oportunidades para que el estudiante pueda irse autoevaluando. De esta manera, se construyen aprendizajes que se van comparando en cada momento respecto a un momento anterior. El orientador resulta fundamental para el intercambio de propuestas, para buscar nuevas estrategias para afrontar otras situaciones de aprendizaje y para la actualización de conocimiento respecto de sus propias fortalezas y debilidades.

\section{Las etapas de la orientación psicoeducativa}

\section{1- La entrevista inicial y el encuadre}


La entrevista inicial, es un instrumento utilizado en la primera sesión del modelo de orientación del CAA-UMCE. Esta tiene el fin de detectar necesidades de acompañamiento, posibilitando que el profesional que realiza la entrevista obtenga información relevante sobre el estudiante, así como también, de la problemática, dificultad o necesidad que lo ha llevado a solicitar ayuda. Si en el área de salud mental se pide que los pacientes revelen algo de sus emociones y su vida personal (Morrison, 2015), en el área educativa, pedimos que el estudiante nos cuente de su trayectoria académica, tratando de explorar cómo sus emociones y su vida personal, afectan sus aprendizajes.

Si bien el foco es en el trayecto recorrido desde que ingresa al sistema de educación formal, establecemos la relación con el contexto en que vive, con las experiencias previas y situaciones personales pasadas y sus emociones actuales. Tal como recomienda Morrison (2015) es importante tratar de obtener la historia y el relato cómo lo siente la persona, esto permitirá tener mayor claridad de las necesidades del estudiante.

Pero ¿qué preguntamos en la primera entrevista? ¿en qué nos enfocamos? Debemos tener claro que el centro es el aprendizaje del estudiante, por lo que las preguntas que realizamos deben dar cuenta de su proceso formativo.

La apertura de la entrevista tampoco debe ser ambigua, pronunciando frases generales o de doble sentido. La entrevista comienza por donde comienza el entrevistado. Hay que tener en cuenta todo lo que puede haberle costado decidir la entrevista y lo que puede significar como factor de humillación y menoscabo para él (Bleger, 1964, p. 18).

Entonces, es importante que comencemos con preguntas abiertas, esto nos permitirá identificar dificultades y las potencialidades que ha tenido durante el trayecto formativo, por ejemplo, indagar en qué le ha costado y que le ha sido fácil al estudiante durante sus estudios secundarios y universitarios o en qué factores lo han ayudado o le han impedido aprobar determinada asignatura. Las dificultades pueden corresponder a etapas anteriores, por ejemplo, durante la educación primaria o secundaria.

Es relevante establecer una aproximación longitudinal y de proceso. Asimismo, es necesario que el estudiante explicite el motivo de consulta, es decir, por qué llegó a solicitar ayuda. Si bien, muchas veces el motivo de consulta no es la raíz del problema, nos permitirá una primera entrada, así como también, negociar los puntos que trabajaremos en las sesiones posteriores. Al final de la sesión, debemos generar acuerdos con el estudiante, de manera de explicitar que los objetivos de trabajo de la próxima sesión. Esto con el fin de que las expectativas del estudiante puedan ser cumplidas.

En la entrevista inicial, estableceremos un encuadre fijo (Bleger, 1964), es decir, instaurar como constantes un conjunto de variables que intervienen en el proceso. El encuadre funciona como una 
especie de estandarización, en el cual se clarifica los roles de cada una de las partes, los objetivos del proceso, el lugar, horario y duración de cada sesión. El encuandre nos permitirá un marco en el cuál la ambigüedad desaparece al explicitar ciertas variables. Si el encuadre es modificado, la variable modificada tiene que ser sujeta a observación, debido a que cada sesión se da en un contexto definido y lo que ocurre ahí solo tiene sentido y significación en relación con dicho contexto.

Es importante que seamos claros sobre la reserva de los datos y antecedentes que proporciona el estudiante, ya que debe existir certeza por parte del estudiante de la confidencialidad de la entrevista y de las sesiones que se desarrollen. Por lo mismo, en caso de solicitud de un informe por parte de la universidad u de un organismo externo, este informe debe ser leído y conocido por el estudiante. Lo mismo ocurre si se va a conversar con docentes u otros profesionales de la institución educativa, el estudiante debe tener conocimiento y estar de acuerdo con esta acción.

Además, en la sesión de entrevista, nos preocuparemos también de crear un ambiente cómodo y acogedor, debemos procurarnos que el estudiante se sienta seguro en este espacio de trabajo. Es nuestra respondabilidad como encargados de la acción, crear un espacio de seguridad donde se compartan experiencias y emociones, y se genere conciencia de las mismas, buscando que se desarrollen habilidades con respecto a las emociones que les son propias, en contextos de confianza y respeto, lo cual facilita la comprensión propia y de los demás.

\section{2- Sobre las sesiones de trabajo de orientación psicoeducativa}

Desde el modelo de orientación psicoeducativa desarrollado en el CAA-UMCE, se trabaja en sesiones de carácter individual o grupal a partir de las distintas necesidades planteadas por el estudiante. Se busca contribuir al desarrollo y potenciación de diversas competencias, con el objeto de mejorar sus procesos de aprendizaje. Estas sesiones se piensan a largo o corto plazo según el objetivo propuesto y negociado.

Para comenzar el trabajo, se utiliza la experiencia personal del estudiante, promoviendo actividades que impliquen vivencias afectivas en un clima de respeto, seguridad y confianza, para fomentar la reflexión desde sus experiencias previas, buscando nuevas posibilidades y construyendo distintas alternativas de abordaje a las situaciones planteadas. La persona que realiza la orientación prepara una serie de actividades, las cuales son planificadas e intencionadas, con el fin de crear contextos que favorezcan la construcción de nuevos aprendizajes.

Es necesario que el estudiante pueda (re)construir relaciones significativas entre las experiencias previas y las competencias que se quieren potenciar, para que sea el propio estudiante el autogestor 
de su propio desarrollo y aprendizaje. Es importante que hagamos preguntas, ejercicios y actividades que permitan el despliegue de ideas del estudiante, pero al mismo tiempo que hagamos visible esos pensamientos, generando productos tangibles. Por ejemplo, Tishman (2002) plantea dos preguntas que puede realizar un docente en una clase: ¿Qué está sucediendo en esta situación? y ¿Qué observas que te lleva a decir eso? Estas mismas preguntas pueden ser utilizadas en la orientación psicoeducativa cuando analizamos una problemática, para facilitar la reflexión sobre la misma. Las respuestas no deben quedarse solamente en eso, sino que deben ser conversadas y visibles a través de una representación concreta, ya sea en una frase escrita o en una imagen. Por ejemplo, escribir un aprendizaje o un pensamiento en una nube de pensamiento.

Si el conocimiento metacognitivo (saber qué) es un conocimiento más explícito y verbalizable, el control metacognitivo (saber cómo) depende de la tarea y el contexto, siendo más implicítico y difícil de verbalizar (Pozo \& Pérez, 2009). En este sentido,

el desarrollo de la metacognición podría avanzar desde un conocimiento más implícito, muy ligado a los conocimientos de un ámbito específico y al contexto de las tareas propias de ese ámbito, hasta el conocimiento más explícito y descontextualizado, que podrían llegar a manifestar los estudiantes más competentes (Pozo \& Pérez, 2009, p.61).

Es necesario un abordaje que fomente el aprendizaje metacognitivo, proponiendo problemáticas que permitan la reflexión sobre distintos momentos del proceso de control metacognitivo. Dependiendo de la situación o problemática planteada se analiza, por ejemplo: con qué medios cuento y qué condiciones tengo, cómo he ido avanzando, cúan lejos o cerca estoy de los objetivos planteado, qué dificultades he tenido para resolverla, qué posibilidades tengo para superar las dificultades, qué medios tengo para mejorar, qué cambios debo realizar.

Es importante realizar ejercicios que permitan la reflexión sobre las situaciones. Si la estudiante plantea que tiene problemas de estrés, vamos a generar ejercicios que permitan que la estudiante reflexione a partir de situaciones concretas. Por ejemplo, la estudiante rindió una prueba la semana pasada. Nosotros podemos trabajar con esa situación. Comenzaremos preparando material concreto que permita la toma de consciencia y que se detenga a analizar esa circunstancia específica. Entregaremos un organizador gráfico que permita analizar rutinas de pensamientos, como el cuadro veo, pienso y me pregunto (figura 1) o el organizador causa-efecto (figura 2). En esta etapa queremos que la estudiante se observe a sí misma e intente comprender su forma de actuar en dicha situación. Esto nos permitirá posteriormente trabajar sobre este actuar, problematizar estos comportamientos, pensamientos y emociones para proponer posibles formas de acción en esa situación, por ejemplo, a través del cuadro que mostramos en la tabla 2. 
Tabla 1: Ejercicio de reflexión metacognitiva sobre una situación específica

\begin{tabular}{cll}
\hline VEO & \multicolumn{1}{c}{ PIENSO } & \multicolumn{1}{c}{ ME PREGUNTO } \\
\hline ¿Qué es lo que observas? & ¿Qué es lo que piensas que & ¿Qué es lo que me preguntó \\
& sucedió? & respecto a lo que sucedió? \\
\hline
\end{tabular}

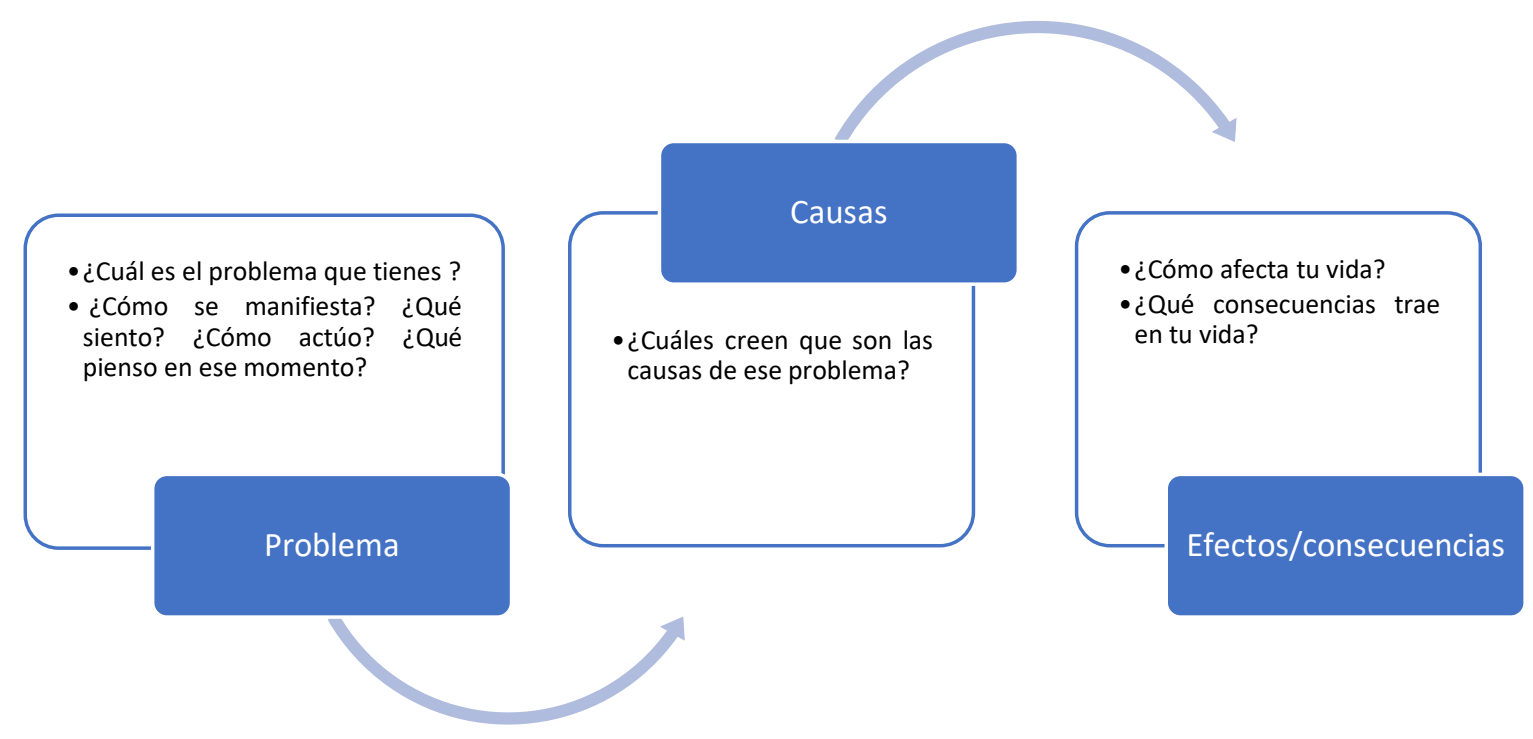

Figura 2. Ejercicio de reflexión sobre una problemática mediante la búsqueda de causas y consecuencias de esta.

Tabla 2: Ejercicio de análisis de comportamientos, pensamientos y emociones que tuve en una situación específica.

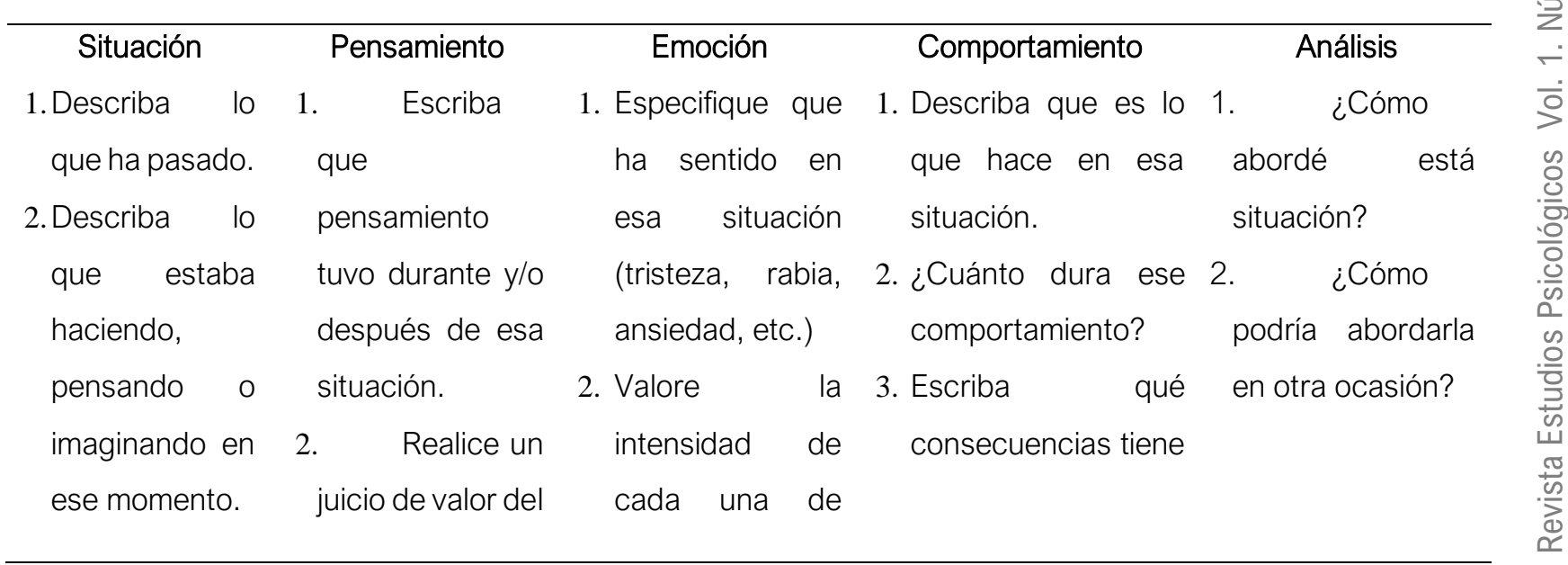




$\begin{array}{llll}\text { pensamiento } & \text { esas emociones } & \text { ese } & \text { 3. ¿Qué } \\ \text { que tuvo (me } & \text { en una escala de } & \text { comportamiento. } & \text { podemos hacer } \\ \text { hace bien, me } & 1 \text { a } 10 & & \text { distinto? } \\ \text { hace mal, etc.) } & & 4 . \quad \text { ¿Cómo } \\ & & \text { podemos cambiar } \\ & \text { nuestros } \\ & \text { comportamientos, } \\ & \text { pensamientos, } \\ & \text { etc.? }\end{array}$

Por otra parte, consideremos relevante en un modelo de orientación, lograr el compromiso del estudiante con su proceso, haciéndolo responsable. Para esto, se desarrolla un trabajo continuo con el estudiante que involucra actividades fuera de sesión, es decir, considera ejercicios en su día a día. De esta manera se traspasa la responsabilidad al estudiante, quien en la interacción cotidiana va (re)construyendo sus significados y significaciones. Los ejercicios que entregamos para realizar fuera de la sesión son concretos y posibilitan visibilizar el pensamiento. Por ejemplo, si a la estudiante le provoca ansiedad una exposición oral, le solicitaremos que lleve una bitácora de registro con una descripción de todas las situaciones en que se ha sentido ansiosa durante la semana. Posteriormente en un diálogo entre el orientador y el estudiante, reflexionamos sobre el ejercicio realizado haciendo consciente diferentes procesos que habitualmente son implícitos. La reflexión del sujeto sobre diferentes experiencias y situaciones y el hacer visible los procedimientos que utiliza permiten ayudar al estudiante a conocer cómo aprende y ejercer control metacognitivo de su propio aprendizaje.

De esta manera, se facilita la generalización y transferencia a otros ámbitos y contextos de aprendizaje, y nos permite que el estudiante adopte estrategias más eficaces para la resolución de problemáticas reemplazando el acercamiento ensayo-error (Pozo \& Pérez, 2009).

\section{3- La evaluación del proceso}

Si abordamos la orientación psicoeducativa desde un enfoque metacognitivo, significa que la evaluación cambiará su foco. En nuestro caso, la enfocamos en el proceso de toma de conciencia del estudiante de sus propios aprendizajes, ya que el orientador será un facilitador del aprendizaje y en la medida en que el estudiante tome consciencia de sus procesos metacognitivos, la orientación será más o menos existosa. Es decir, el énfasis no estará puesto en el resultado final, sino en los aprendizajes que realiza el estudiante sesión a sesión y que son visibles para él. 
A través de la realización de actividades concretas (por ejemplo, resolución de rompecabezas o un problema planteado) que son elegidas según los objetivos y su posterior análisis, trabajaremos la metacognición, toma de consciencia e introspección. Al final de la sesión, propondremos un ejercicio final que ayude al estudiante a generar síntesis de los aprendizajes de esa sesión. Este ejercicio debe permitir que el estudiante vea sus pensamientos reflejados en un producto concreto, y a su vez, le permitan reflexionar sobre si ha alcanzado el objetivo propuesto para la sesión y a qué se puede atribuir la consecución o la no consecución del objetivo. Esta misma evaluación que realizamos sesión a sesión permitirá al orientador, evaluar el proceso de orientación que está realizando con el estudiante y mejorarlo en caso de ser necesario. Asimismo, en la última sesión que se tenga, debemos realizar una evaluación de todo el proceso de orientación, que permita su cierre. Para esto, podemos trabajar con líneas de tiempo, diagramas de flujo, mapas mentales, entre otros. Lo importante es que esta evaluación pueda plasmarse en un trabajo que el estudiante pueda conservar, para que pueda observar y reflexionar sobre el proceso en otros espacios.

Entrevista inicial con preguntas abiertas para detectar necesidades e identificar potencialidades.

- Establecer un encuadre (definir roles, establecer lugar, horario y duración de la sesión).

- Crear un espacio de seguridad y confiaza.

- Explicitar criterio de

confidencialidad de la información.

- Negociar acuerdos y objetivo de

trabajo con el estudiante.

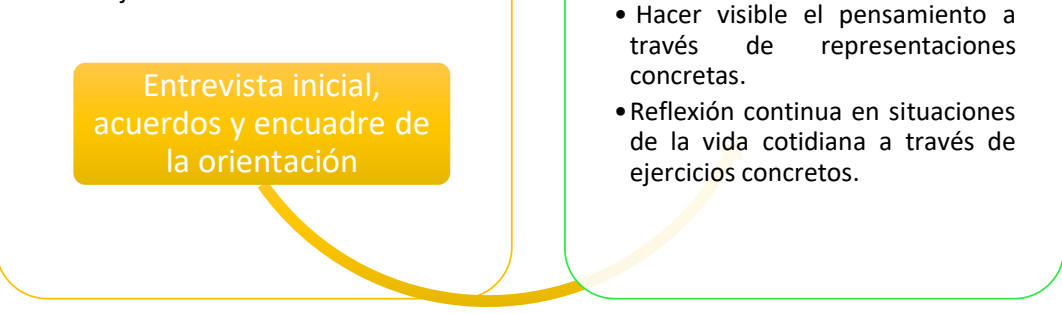

Sesiones de orientación

\section{psicoeducativa}

- En función de las necesidades del estudiante.

- Duración del proceso depende de los objetivos propuestos

- Actividades intecionadas y planificadas que buscar la reflexión metacognitiva.

- Hacer visible el pensamiento a través de representaciones concretas.

Reflexión continua en situaciones de la vida cotidiana a través de ejercicios concretos.
- La evaluación se realiza sesión a sesión

- Ejercicios metacognitivos que permiten hacer visible $y$ ser conciente de mis aprendizajes.

- Evaluación final sobre el proceso que se plasma en un producto que el estudiante genera y conserva.

\section{Evaluación de proceso}

Figura 2: Etapas de la orientación psicoeducativa

Fuente: Elaboración propia

\section{CONCLUSIONES}

La orientación psicoeducativa constituye una estrategia de acompañamiento que permite la inclusión y el trabajo con distintos sujetos en función de sus propias necesidades de aprendizaje. Un modelo 
de orientación psicoeducativa debe en primer lugar definir el foco del proceso de orientación psicoeducativa, es decir, el qué, el cómo y el para qué de la orientación.

Cuando el centro de la orientación es la mejora de los aprendizajes de un sujeto estudiante universitario, todas las prácticas son elaboradas pensando en a) cómo potenciar los procesos de aprendizaje y b) en el estudiante cómo protagonista de la acción. Por lo anterior, se vuelve relevante conocer qué estrategias utiliza el estudiante en su proceso de aprendizaje y cuál ha sido su trayectoria educativa. En nuestro caso, utilizamos como instrumento para indagar en los puntos anteriores una entrevista inicial, en la cual se realiza el encuadre y se negocian los objetivos del proceso de orientación. La negociación de los objetivos del proceso, la retroalimentación del estudiante y la reflexión sobre el proceso sesión a sesión, son fundamentales en la orientación psicoeducativa a nivel universitario, debido a que es importante fomentar la construcción de un profesional crítico y reflexivo.

Otro elemento que debemos tener en cuenta es el nivel educativo en que se desarrolla la orientación, en este caso, educación superior universitaria. Cada licenciatura exige el desarrollo de ciertas competencias transversales y específicas que es importe conocer, pues muchas veces, es posible trabajarlas en conjunto con los estudiantes y potenciarlas. Asimismo, es importante conocer quién es el estudiante, saber sus características sociodemográficas, su trayectoria académica, sus intereses, gustos y disgustos, su vida cotidiana en la universidad, pues el proceso de orientación psicoeducativa acordado con el estudiante debe estar diseñado según las necesidades de éste, ser pertinente a las problemáticas planteadas y a su trayectoria académica y apuntar a un desarrollo integral.

En la entrevista inicial se recaba la mayor cantidad de información. Esto permitirá ajustar el proceso al sujeto con que trabajamos. Asimismo, podremos triangular la información con otras aportadas por registros académicos y otros actores de la institución educativa. Es importante tener presente que somos un actor más en el proceso de acompañamiento, por lo que trabajar en red con otros departamentos, docentes, profesionales permitirá potenciar el proceso de orientación, beneficiando al estudiante.

Posteriormente, deberemos diseñar la primera sesión en concordancia con las necesidades planteadas, pero considerando sus intereses. Las sesiones siguientes, se planifican, a partir del desarrollo y retroalimentación de cada sesión, por lo que es el proceso será dinámico y flexible, pudiendo modificarse, y en muchos casos, debiendo renegociarse el objetivo inicial de orientación durante el proceso. 
Asimismo, debemos facilitar la creación de redes entre los distintos actores de la comunidad universitaria que brindan acompañamiento a los estudiantes. Esto nos otorgará fluidez en el proceso y permitirá aprovechar los recursos existentes en la institución, mejorando las problemáticas que devienen del proceso educativo.

\section{REFERENCIAS}

Arraiz, A. \& Sabirón, P. (2012). Orientación para el aprendizaje a lo largo de la vida: modelos y tendencias. Zaragoza: Prensas de la Universidad de Zaragoza

Barrientos, A. (2016). Habilidades sociales y emocionales del profesorado de educación infantil relacionadas con la gestión del clima de aula. Tesis para optar al grado de doctor. Madrid: Universidad Complutense de Madrid. https://eprints.ucm.es/40450/1/T38117.pdf

Bisquerra, R. (2003). Educación emocional y competencias básicas para la vida. Revista de Investigación Educativa (RIE), 21, 1, 7-43.

Bisquerra, R. (2009). Psicopedagogía de las emociones. Madrid: Síntesis.

Bleger, J. (1964). La entrevista Psicológica. Buenos Aires: Nueva Visión.

Chantharanuwong, W., Thatthong, K., Yuenyong, C. \& Thomas, G. (2012), Exploring the Metacognitive Orientation of the Science Classrooms in a Thai Context, Procedia - Social and Behavioral Sciences, 46, 5116-5123. https://doi.org/10.1016/j.sbspro.2012.06.393

Del Pino, J. (2013). Orientación educativa y profesional en el contexto cubano: concepciones, experiencias y retos. Revista Alternativas Cubanas en psicología, 1 (2), 48-54. https://www.acupsi.org/articulo/29/orientacin-educativa-y-profesional-en-el-contexto-cubanoconcepciones-experiencias-y-retos.html

Espinoza, O. \& González-Fiegehen, L. (2015). Equidad en el Sistema de Educación Superior de Chile: Acceso, Permanencia, Desempeño y Resultados. Santiago de Chile: Ceppe.

Garbanzo, G. (2007). Factores asociados al rendimiento académico en estudiantes universitarios, una reflexión desde la calidad de la educación superior pública. Educación, 31(1), 43-63.

Jaramillo, L. \& Simbaña, V. (2014). La metacognición y su aplicación en herramientas virtuales desde la práctica docente. Sophia, Colección de Filosofía de la Educación, (16),299-313. https://www.redalyc.org/pdf/4418/441846097014.pdf 
Jarpa, C. \& Rodríguez, C. (2017). Segmentación y exclusión en Chile: El caso de los Jóvenes Primera Generación en Educación Superior. Revista Latinoamericana de Ciencias Sociales, Niñez y Juventud, 15(1),327-343. https://www.redalyc.org/pdf/773/77349627021.pdf

Llanos, M. \& Martínez, A. (2018). Diseño e Implementación de un Modelo de Gestión en Docencia Universitaria: Caso Carreras de Ingeniería de la Universidad Arturo Prat, Chile. Formación universitaria, 11(6), 3-16. https://dx.doi.org/10.4067/S0718-50062018000600003

Morrison, J. (2015). La entrevista psicológica. México, D.F.: Editorial El Manual Moderno.

Nieves, Z., Otero, I., \& Molerio, O. (2006). La formación profesional en la universidad de hoy: de la educación a la autoeducación. Revista Iberoamericana De Educación, 39(6), 1-8. https://rieoei.org/historico/deloslectores/1480Achon.pdf

Núñez, J., Solano, P, González-Pienda, J., \& Rosário, P. (2006). El aprendizaje autorregulado como medio y meta de la educación. Papeles del Psicólogo, 27(3),139-146. https://www.redalyc.org/pdf/778/77827303.pdf

Pozo, J. \& Pérez, M. (2009). Psicología del aprendizaje universitario: La formación en competencias. Madrid: Ediciones Morata.

Sánchez, P. (2017). La orientacion educativa en la universidad desde la perspectiva de los

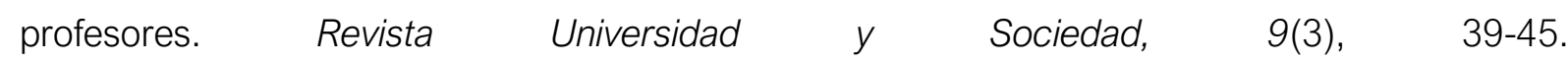
http://scielo.sld.cu/scielo.php?script=sci_arttext\&pid=S2218-36202017000300006

Saarni C., 2000, Emotional competence: A developmental perspective. In R. Bar-On \& J. D. Parker (Eds.), The handbook of emotional intelligence. San Francisco: Jossey-Bass, 68-91.

Schiefelbein, E. y Zuñiga, R. (2000). Relaciones de la Educación Superior con la Educación Secundaria: transformación de la enseñanza, investigación y extensión universitarias. Seminario sobre Prospectiva Educativa. Santiago: UNESCO. https://www.schwartzman.org.br/simon/delphi/

Serrano, J. M. y Pons, R. M. (2011). El constructivismo hoy: enfoques constructivistas en educación. Revista Electrónica de Investigación Educativa, 13(1). http://redie.uabc.mx/vol13no1/contenidoserranopons.html

Tishman, S. (2002). Artful reasoning. In Grotzer, T., Howick, L., Tishman, S. \& Wise, D., Art works for schools: A curriculum for teaching thinking in and through the arts. Lincoln, MA: DeCordova Museum and Sculpture Park. 
UMCE (2016). Modelo Educativo Universidad Metropolitana de Ciencias de la Educación. Santiago:

Universidad

Vera, L. (2014). La autonomía del estudiante dentro del espacio social y académico en la Universidad Veracruzana. Las dimensiones y regulaciones disciplinarias. Ciencia Huasteca Boletín Científico De La Escuela Superior De Huejutla, 2(4). https://doi.org/10.29057/esh.v2i4.1073 